\title{
Ultraviolet to mid-infrared gas-filled anti-resonant hollow-core fiber lasers
}

\author{
Wang, Yazhou; Adamu, Abubakar I.; Habib, Md Selim; Dasa, Manoj K.; Petersen, Christian R.; Antonio \\ Lopez, J. Enrique; Zhou, Binbin; Jepsen, Peter Uhd; Schülzgen, Axel; Bache, Morten
}

Total number of authors:

13

Published in:

Proceedings of SPIE

Link to article, DOI:

10.1117/12.2592717

Publication date:

2021

Document Version

Publisher's PDF, also known as Version of record

Link back to DTU Orbit

Citation (APA):

Wang, Y., Adamu, A. I., Habib, M. S., Dasa, M. K., Petersen, C. R., Antonio Lopez, J. E., Zhou, B., Jepsen, P. U., Schülzgen, A., Bache, M., Correa, R. A., Bang, O., \& Markos, C. (2021). Ultraviolet to mid-infrared gas-filled anti-resonant hollow-core fiber lasers. In K. Kalli, A. Mendez, \& P. Peterka (Eds.), Proceedings of SPIE (Vol. 11773). [1177308] SPIE - International Society for Optical Engineering. Proceedings of SPIE - The International Society for Optical Engineering Vol. 11773 https://doi.org/10.1117/12.2592717

\section{General rights}

Copyright and moral rights for the publications made accessible in the public portal are retained by the authors and/or other copyright owners and it is a condition of accessing publications that users recognise and abide by the legal requirements associated with these rights.

- Users may download and print one copy of any publication from the public portal for the purpose of private study or research.

- You may not further distribute the material or use it for any profit-making activity or commercial gain

- You may freely distribute the URL identifying the publication in the public portal 


\section{Ultraviolet to mid-infrared gas-filled anti-resonant hollow-core fiber lasers}

Wang, Yazhou, Adamu, Abubakar, Habib, Md. Selim, Dasa, Manoj, Petersen, Christian, et al.

Yazhou Wang, Abubakar I. Adamu, Md. Selim Habib, Manoj K. Dasa, Christian R. Petersen, J. Enrique Antonio-Lopez, Binbin Zhou, Peter Uhd Jepsen, Axel Schülzgen, Morten Bache, Rodrigo Amezcua Correa, Ole Bang, Christos Markos, "Ultraviolet to mid-infrared gas-filled anti-resonant hollowcore fiber lasers," Proc. SPIE 11773, Micro-structured and Specialty Optical Fibres VII, 1177308 (18 April 2021); doi: 10.1117/12.2592717 


\title{
Ultraviolet to mid-infrared gas-filled anti-resonant hollow-core fiber lasers
}

\author{
Yazhou Wang ${ }^{* a}$, Abubakar I. Adamu ${ }^{\mathrm{a}}$, Md Selim Habib ${ }^{\mathrm{b}, \mathrm{c}}$, Manoj K. Dasa ${ }^{\mathrm{a}}$, Christian \\ R. Petersen ${ }^{\mathrm{a}, \mathrm{e}}$, J. Enrique Antonio Lopez, Binbin Zhou ${ }^{\mathrm{a}}$, Peter Uhd Jepsen ${ }^{\mathrm{a}, \mathrm{e}}$, Axel \\ Schülzgen $^{\mathrm{b}}$, Morten Bache ${ }^{\mathrm{a}}$, Rodrigo Amezcua Correa ${ }^{\mathrm{b}}$, Ole Bang, ${ }^{\mathrm{a}, \mathrm{e}}$, and Christos Markos ${ }^{\mathrm{a}, \mathrm{e}}$ \\ ${ }^{a}$ DTU Fotonik, Technical University of Denmark, DK-2800 Kgs. Lyngby, Denmark. \\ ${ }^{\mathrm{b}}$ CREOL, The College of Optics and Photonics, University of Central Florida, Orlando, FL-32816 \\ USA. \\ 'Department of Electrical and Computer Engineering, Florida Polytechnic University, Lakeland, \\ Florida 33805, USA. \\ dNKT Photonics, Blokken 84, DK-3460 Birkerød, Denmark. \\ eNORBLIS IVS, Virumgade 35D, DK-2830 Virum, Denmark.
}

\begin{abstract}
We will present our recent works on fiber lasers enabled by noble and Raman-active gas-filled anti-resonant hollow-core fiber (ARHCF) technology. First, we will present the generation of supercontinuum (SC) spanning from $200 \mathrm{~nm}$ to $4 \mu \mathrm{m}$ based on a Argon (Ar)-filled ARHCF pumped at $2.46 \mu \mathrm{m}$ wavelength with $100 \mathrm{fs}$ pulses and $\sim 8 \mu \mathrm{J}$ pulse energy. Then we will discuss our recent work on stimulated Raman scattering (SRS) effect in a hydrogen $\left(\mathrm{H}_{2}\right)$-filled ARHCF, to achieve infrared Raman lasers. By employing the single-stage vibrational SRS effect, a $4.22 \mu \mathrm{m}$ Raman laser line is directly converted from a linearly polarized $1.53 \mu \mathrm{m}$ pump laser. A quantum efficiency as high as $74 \%$ was achieved, to yield 17.6 $\mu \mathrm{J}$ pulse energy. The designed $4.22 \mu \mathrm{m}$ wavelength is overlapped with the strongest $\mathrm{CO}_{2}$ absorption, therefore constituting a promising way for $\mathrm{CO}_{2}$ detection. In addition, we report a multi-wavelength Raman laser based on cascaded rotational SRS effect. Four Raman lines at $1683 \mathrm{~nm}, 1868 \mathrm{~nm}, 2099 \mathrm{~nm}$, and $2394 \mathrm{~nm}$ are generated, with pulse energies as high as $18.25 \mu \mathrm{J}, 14.4 \mu \mathrm{J}, 14.1 \mu \mathrm{J}$, and $8.2 \mu \mathrm{J}$, respectively. The energy of these Raman lines can be controlled by tuning the $\mathrm{H}_{2}$ pressure from 1 bar to 20 bar.
\end{abstract}

Keywords: Anti-resonant hollow-core fiber, supercontinuum, Raman laser.

\section{INTRODUCTION}

The recent advent of silica ARHCF technology has enabled new research directions towards development of novel lasers from deep-ultraviolet (DUV) to the mid-infrared (MIR) ${ }^{1}$. The laser beam is confined within the hollow-core region of ARHCF through anti-resonant effect, therefore possessing important advantages of gas medium such as low loss, broad transmission range, and high damage threshold. The easy control of gas pressure and species allows the flexible tailoring of fiber nonlinearity and dispersion properties. These factors make the ARHCF an ideal platform for the propagation of high peak power and energy laser pulse with rich nonlinear dynamics such as four-wave mixing ${ }^{2}$, pulse compression ${ }^{3}$, and resonant dispersive wave (DW) emission ${ }^{4}$. An influential result of these nonlinear effects is the SC generation broadly spanning from DUV to MIR spectral region ${ }^{5}$. In 2019, we reported that pumping a $30 \mathrm{~cm}$ long Ar-filled ARHCF under 30 bar pressure with a $2460 \mathrm{~nm}$ laser with $\sim 100 \mathrm{fs}$ pulse duration and $\sim 8 \mu \mathrm{J}$ pulse energy, to generate a SC from $200 \mathrm{~nm}$ up to $4 \mu \mathrm{m}^{6}$. Further investigations have been performed in recent to achieve a tunable DUV SC by pumping an Ar-filled ARHCF with a $1030 \mathrm{~nm}$ laser with a pulse energy of $24.2 \mu \mathrm{J}$ and compressed pulse duration of $30 \mathrm{fs}^{7}$. As a result, the center wavelength of DW can be tuned from 236 to $377 \mathrm{~nm}$ by adjusting the Ar pressure from 2.2 to 5.7 bar.

SRS is another important nonlinear effect which occurs as a result of the intense interaction between laser pulse and a Raman active gas-filled in ARHCF ${ }^{8,9}$. The SRS effect leads to the emission of Stokes and anti-Stokes waves, and are widely used for laser frequency down- and up-shifts. Phase matching condition of the anti-Stokes emission can be satisfied with the pressured dependent fiber dispersion property and the dispersion difference of different order modes ${ }^{10,11}$. Contrary to SC generation, the gas-filled fiber Raman laser can achieve a narrow linewidth in few GHz level and even hundreds $\mathrm{MHz}$ level ${ }^{11-13}$. This is because SRS process in gas has a narrow Raman gain bandwidth ${ }^{14}$, and can occur at a lower laser intensity compared to other nonlinear effects such as self-phase modulation (SPM). By employing vibrational SRS effect

Micro-structured and Specialty Optical Fibres VII, edited by Kyriacos Kalli, Alexis Mendez, Pavel Peterka, Proc. of SPIE Vol. 11773, 1177308 · @ 2021 SPIE · CCC code: 0277-786X/21/\$21 · doi: 10.1117/12.2592717 
in gases such as $\mathrm{H}_{2}$ and methane $\left(\mathrm{CH}_{4}\right)$ with a long Stokes frequency shift coefficient ${ }^{15}$, MIR single-wavelength laser can be directly converted from the mature near-infrared (NIR) region. Several impressive reports on MIR fiber Raman lasers have already been reported, based on the vibrational SRS process in $\mathrm{H}_{2}$ and $\mathrm{CH}_{4}{ }^{16-19}$. In these reports, the quantum efficiency can easily reach a desirable level (e.g., $>60 \%$ ), leading to high pulse energies in micro-joule range. Multiwavelength (or frequency comb-like) Raman lasers due to cascaded (anti-) Stokes effects have also been reported 9,18,20-22, which have versatile applications such as sensing, wavelength division multiplexing communication, and optical signal processing ${ }^{23}$. In comparison with the conventional method of selecting multiple wavelengths with a comb filter within the limited gain bandwidth of a rare-earth ion-doped gain medium ${ }^{24}$, this method can achieve multi-wavelength operation in a much broader wavelength range.

In this paper, we present our rent works regarding gas-filled ARHCF lasers, including a SC generation from $200 \mathrm{~nm}$ to $4 \mu \mathrm{m}$, a MIR Raman laser generation at $4.22 \mu \mathrm{m}$ with high energy of $17.6 \mu \mathrm{J}$ and $4.22 \mu \mathrm{m}$, and a multi-wavelength Raman laser spanning from $1.53 \mu \mathrm{m}$ to $2.4 \mu \mathrm{m}$.

\section{SC GENERATION BASED ON AR-FILLED ARHCF}

Figure 1(a) shows the experimental setup of SC generation. A Ti:sapphire amplifier was used to pump an OPA with a few milijoule pulse energy to generate a MIR pump. The MIR pump is a linearly polarized $100 \mathrm{fs}$ pulses at $1 \mathrm{kHz}$ repetition rate with a central wavelength of $2460 \mathrm{~nm}$ and its power was controlled by neutral density (ND) filters and by rotating a nanoparticle linear film polarizer (P1). The pump laser is coupled into a $30 \mathrm{~cm}$ long ARHCF through a planoconvex lens with $5 \mathrm{~cm}$ focal length. Both ends of the ARHCF are sealed by two gas cells for Ar filling. Optical windows and lenses used in this setup were uncoated $\mathrm{CaF}_{2}$ to obtain $>90 \%$ transmission from 200-5000 nm. The laser at the output of the fiber is collimated and directed to either a CCD-based detector (Oceanoptics HR2000+) or to an infrared Spectrometer by using a flip-mirror, to measure the output spectrum at 183-1100 nm and 1000-5000 nm, respectively.

The ARHCF consists of seven non-touching silica capillaries with wall thickness of $\sim 640 \mathrm{~nm}$ forming a core with diameter of $\sim 44 \mu \mathrm{m}$, as shown in the scanning electron microscope (SEM) images of Figure 1(a). The propagation loss of the ARHCF is simulated based on a finite element method (FEM) using Comsol software with contributions from the material (silica), mode confinement, and surface scattering loss. According to the simulation result in Figure 1(b), one can see that the ARHCF transmits light over a broad spectral range with the anti-resonant window spanning from $\sim 1400 \mathrm{~nm}$ to $\sim 4000 \mathrm{~nm}$, which is consistent with the measured loss spectrum based on cut-off method. Higher order anti-resonant windows allow for light to propagate even in the DUV range close to the bandgap of fused silica. The refractive index used in the calculations was based on the Sellmeier equation found in Ref. [25], which is valid from $210 \mathrm{~nm}$ up to $3.7 \mu \mathrm{m}$. However, it is important to mention that careful consideration of the silica refractive index is required for wavelengths less than $210 \mathrm{~nm}$ due to strong electronic bandgap absorption ${ }^{26}$. To this end, the measured refractive index data of silica from Ref. [26] was used to calculate the GVD and confinement loss for wavelengths less than $210 \mathrm{~nm}$. Figure 1(c) shows the calculated GVD of the fiber from $200 \mathrm{~nm}$ to $3700 \mathrm{~nm}$ for different gas pressures from 0 to $30 \mathrm{bar}$. When the pressure of the gas in the fiber increases it counterbalances the anomalous dispersion of the fiber, and as a result the ZDW shifts towards longer wavelengths and the nonlinear refractive index $\mathrm{n}_{2}$ increases, as it has been extensively described elsewhere $1,27,28$.

Before the start of the experiments, the fiber was purged several times with high purity Ar to remove any remaining atmospheric air and other impurities. Initial increase of the pump power is associated with the SPM dominated spectrum broadening, which is found to be weakly dependent on pressure. When the spectrum broadens to visible region, it becomes pressure sensitive due to the significant change of the GVD in the visible compared to the MIR. The pressure have a more pronounced effect on the MIR and UV-visible regime at higher pump pulse energy. At the maximum pulse energy of 20 $\mu \mathrm{J}$, the increase of Ar pressure first leads to significant broaden toward UV region with a dispersive wave at $240 \mathrm{~nm}$. When the pressure is above 15 bar the long-wavelength edge starts to quickly increase, meanwhile the energy of the other DW around $275 \mathrm{~nm}$ slowly rises up to replace the DWs around $240 \mathrm{~nm}$. The increasing MIR broadening and $275 \mathrm{~nm}$ DW generation is attributed to the increasing nonlinearity, while the diminishing $240 \mathrm{~nm}$ DW generation is believed to be caused by the red-shifting of the ZDW. Figure 1(d) shows the measured SC spectrum spanning from $200 \mathrm{~nm}$ up to $4 \mu \mathrm{m}$ at the maximum pulse energy of $20 \mu \mathrm{J}$. The main limitation of the spectral extension towards the MIR is the increasing propagation losses, reaching $100 \mathrm{~dB} / \mathrm{m}$ at $3500 \mathrm{~nm}$ from which silica multiphonon absorption is the dominant loss mechanism. To improve the MIR transmission it is therefore paramount to reduce the mode-filed overlap with the silica structure to a minimum, which can be achieved through fiber geometric modification. 

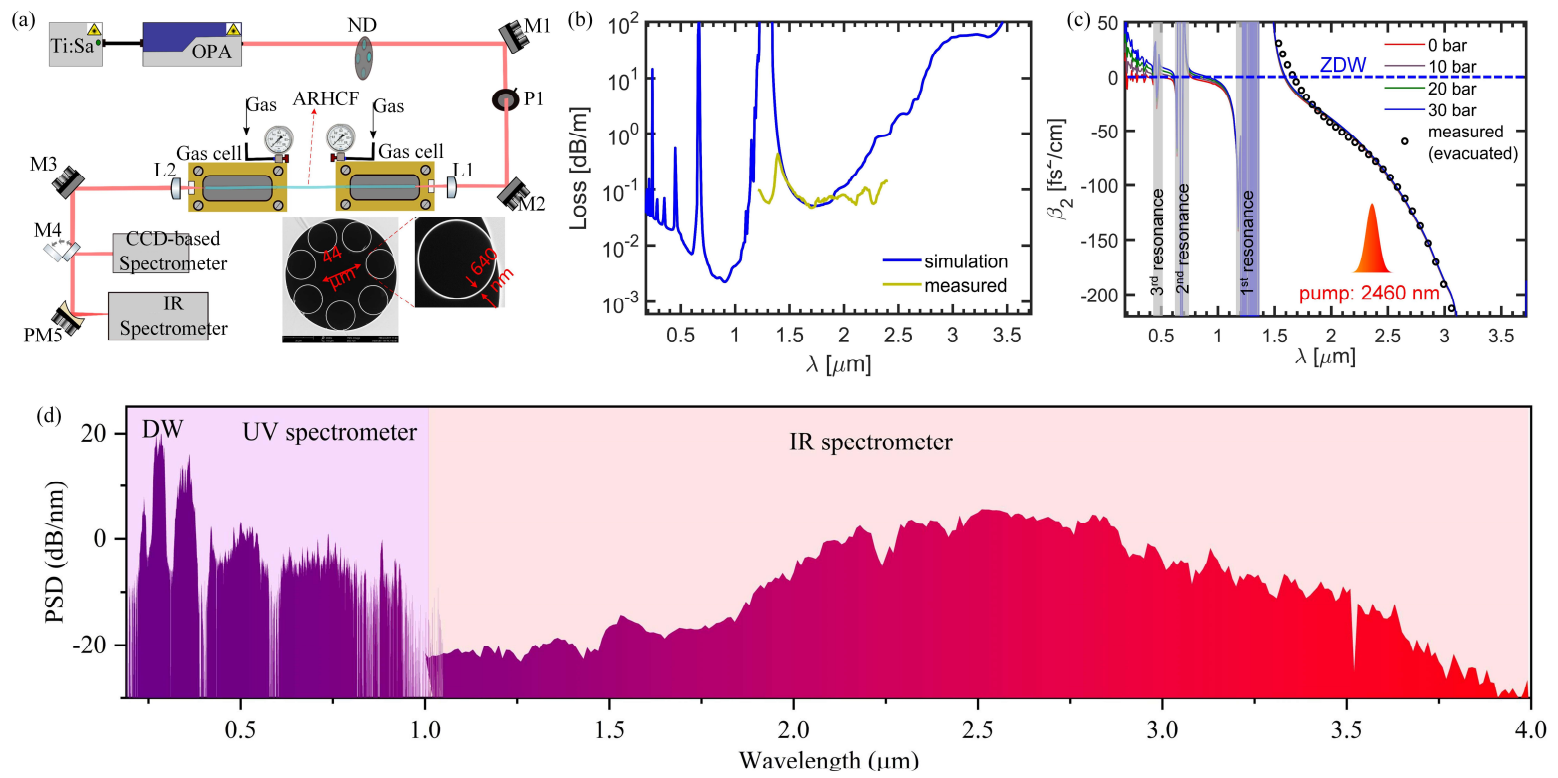

Figure 1. SC generation based on Ar-filled ARHCF. (a) Experimental setup. Insets in bottom are SEM images of the ARHCF. The components of the setup are: neutral density filter (ND), protected silver mirrors (M1-2), linear film polarizer ( $\mathrm{P} 1), \mathrm{CaF}_{2}$ plano-convex lenses (L1-2), flip mirror (F1), and a gold coated parabolic mirror (PM1), $30 \mathrm{~cm}$ of ARHCF. (b) Simulated losses (blue) of the ARHCF, including contributions from surface scattering, mode confinement, and silica material loss. A measured loss spectrum based on cut-off method is provided for comparison. (c) Calculated group velocity dispersion (GVD) for the ARHCF at different levels of Ar pressure (solid lines). The measured dispersion of the fiber in the MIR is shown in circles for comparison (measured at ambient air condition). (d) Measured spectrum of SC obtained by pumping the ARHCF with $100 \mathrm{fs}$ pulses at $2460 \mathrm{~nm}$ (anomalous dispersion region).

\section{MIR RAMAN HIGH NERGY RAMAN LASER GENERATION}

Figure 2(a) shows the experimental setup of Raman laser generation. The pump laser is a master oscillator power amplifier (MOPA) based on a directly modulated distributed feedback diode laser. The seed oscillator delivers a linearly polarized pulse train at a repetition rate of $8 \mathrm{kHz}$ with $6.9 \mathrm{~ns}$ pulse duration. The center pump wavelength was measured to be $1532.8 \mathrm{~nm}$, corresponding to a Raman laser wavelength of $4.22 \mu \mathrm{m}$. The seed laser is first pre-amplified using two stages of core-pumped Er-doped fiber (EDF) pre-amplifiers sharing the same $\mathrm{CW}$ pump laser diode. The final amplification stage is a co-doped double-cladded $\mathrm{Er} / \mathrm{Yb}$ fiber cladding-pumped by a $10 \mathrm{~W} \mathrm{CW}$ laser diode at $915 \mathrm{~nm}$. The large core size of $25 \mu \mathrm{m}$ of the Er:Yb-doped fiber effectively suppresses the laser linewidth broadening from nonlinear effects. Band pass filters with $\sim 2 \mathrm{~nm}$ bandwidth were used to suppress the ASE after each amplifier. After the power amplifier, the maximum average power was measured to be $1.41 \mathrm{~W}$ (including ASE). According to the spectral intensity distribution shown in Figure 2(a), the average power of the pulse signal is estimated to be $643 \mathrm{~mW}$, corresponding to 11.6 $\mathrm{kW}$ peak power and $80.4 \mu \mathrm{J}$ pulse energy. The polarization extinction ratio (PER) of the pump laser is measured to be -19 $\mathrm{dB}$, which includes the contributions of both the pulse signal and the ASE. Because the ASE generated during the amplification process is intrinsically un-polarized, the pulse signal in isolation is expected to have higher PER. The laser linewidth was measured by an optical spectrum analyzer (ANDO AQ6317B, AssetRelay) with a resolution of $0.01 \mathrm{~nm}$ to be $0.06 \mathrm{~nm}$.

$\mathrm{H}_{2}$ is filled into a piece of ARHCF using two gas cells. A half-wave plate (HWP) is placed before the ARHCF to optimize the laser polarization direction and maximize the Raman laser power ${ }^{29}$. The output beam from the pump laser was collimated and coupled into the ARHCF through a pair of plano-convex lenses. The coupling efficiency was $\sim 81 \%$. At the output side of the ARHCF, a $2.4 \mu \mathrm{m}$ long-pass filter with $20 \%$ transmission loss at $4.22 \mu \mathrm{m}$ is used to extract the vibrational Raman line laser from the residual pump. Figure 2(b) shows the SEM images of the ARHCF. The cladding consists of a ring of 7 capillaries with a wall thickness of $1030 \mathrm{~nm}$ and a diameter of $43.6 \mu \mathrm{m}$, forming an air-core region with a diameter of $\sim 73 \mu \mathrm{m}$. This structure enables two transmission bands separated by a strong loss peak centered at 2.0 $\mu \mathrm{m}$, as can be seen in the simulated loss spectrum depicted in Figure 2(c). The simulation was done based on the finiteelement method using software COMSOL with parameter settings identical to those in Ref. [30], but without taking into 
consideration the surface scattering loss due to the small surface roughness of the silica waveguide in the infrared region and the large core diameter of the fiber ${ }^{31}$. The measured loss at $1.53 \mu \mathrm{m}$ is $\sim 0.05 \mathrm{~dB} / \mathrm{m}$ obtained via a cut-back method using the pump laser as light source. The fiber loss in the 2.5-4.5 $\mu \mathrm{m}$ region was also measured with a cut-back method, but using a broadband MIR SC source (MIR-SuperK, NKT Photonics). The loss at $4.22 \mu \mathrm{m}$ could not be precisely obtained by the cut-back measurement because of the $\mathrm{CO}_{2}$ presence in the ambient air inside the fiber, but from the surrounding background loss we can see it is approximately $1.5 \mathrm{~dB} / \mathrm{m}$. Note that the loss difference between the simulation and experiment slowly increases toward shorter wavelengths. This effect is due to the wavelength-dependent bend loss of the fiber (see Eq. (4) in Ref. [32], where the minimum bend radius is inversely proportional to the square of wavelength).

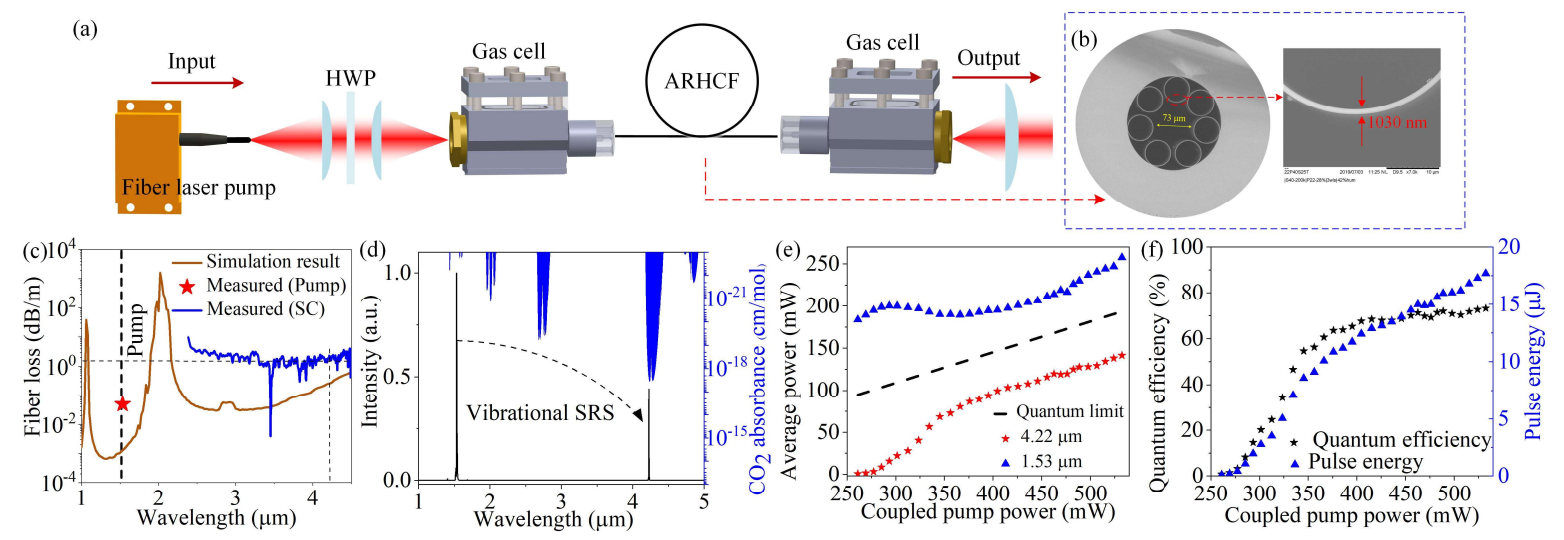

Figure 2. MIR Raman laser generation in $\mathrm{H}_{2}$-filled ARHCF. (a) Experimental setup. (b) SEM images of the ARHCF. (c) Simulated (brown curve) and measured (blue curve) loss spectra of the ARHCF. The red star indicates the measured loss at the pump wavelength of $1532.8 \mathrm{~nm}$ using the cutback method. (d) Spectra of pump and Raman laser. Top axis shows the absorbance spectrum of $\mathrm{CO}_{2}$ obtained from the high-resolution transmission molecular absorption database (HITRAN). (e) Evolution of average Raman laser power and residual pump power as a function of coupled pump power. The dash line shows the quantum limit of the Raman laser. (f) Calculated quantum efficiency and pulse energy corresponding to (e).

The length of the ARHCF was optimized to $3.95 \mathrm{~m}$, to maximize the Raman laser power at the maximum pump power. In this condition, it is found that the Raman power scales up with increasing $\mathrm{H}_{2}$ pressure and steadily reaches saturation power at $\sim 141 \mathrm{~mW}$ when the pressure approaches 20 bar. This trend can be attributed to the known saturation of the Raman gain at high pressure and decreased dephasing time of $\mathrm{H}_{2}{ }^{33}$. Figure 2(d) depicts the spectrum at 20 bar measured using an optical spectrometer (Spectro 320, Instrument Systems) with a resolution of $0.05 \mathrm{~nm}$, where a strong laser line at $4.22 \mu \mathrm{m}$ is formed as a result of the vibrational Raman Stokes shift from the pump laser. A rotational Raman line is also formed at $1.68 \mu \mathrm{m}$, but its intensity is negligible when compared to the vibrational Raman line as a result of the optimized pump polarization ${ }^{34}$. Simulated absorbance spectrum of $\mathrm{CO}_{2}$ is also provided for comparison in this figure. It can be seen that the spectrum of Raman laser is well located in the R-branch of absorption spectrum of $\mathrm{CO}_{2}$ molecules, suggesting the great usability of this laser for high sensitivity $\mathrm{CO}_{2}$ monitoring. The dependences of the Raman laser power and residual pump power on the coupled pump power are measured and shown in Figure 2(e). By increasing the coupled pump power, the power of the Raman laser continuously increases while its growth rate gradually decreases to a nearly constant value. Correspondingly, the calculated quantum efficiency in Figure 2(f) becomes nearly independent on the pump power after $\sim 70 \%$. At $20 \mathrm{bar}$, the maximum quantum efficiency is $74 \%$, corresponding to $17.6 \mu \mathrm{J}$ pulse energy. To the best of our knowledge, both pulse energy and quantum efficiency here are the highest compared to all reported gasfilled ARHCF lasers beyond $3 \mu \mathrm{m}$, including both fiber laser and solid-state laser pumping.

\section{MULTI-WAVELENGTH NIR RAMAN LASER GENERATION}

Besides the presented vibrational Raman laser in last section, the rotational SRS process of $\mathrm{H}_{2}$ can also be adopted for laser frequency shift. The efficiency of rotational SRS can be significantly enhanced by simply changing the pump polarization from linear to circular state ${ }^{34}$. Due to its short frequency shift coefficient of $587 \mathrm{~cm}^{-1}$ and the wide transmission band of ARHCF, this property is suitable for cascaded Raman laser generation with multiple wavelengths. Here we demonstrate a high pulse energy multi-wavelength Raman laser spanning from $1.53 \mu \mathrm{m}$ up to $2.4 \mu \mathrm{m}$ by replacing the above ARHCF with a $5 \mathrm{~m}$ ling nested ARHCF. The HWP is replaced by a quarter-wave plate before the nested ARHCF to convert the pump light from linear polarization to circular polarization and to optimize the polarization orientation. The 
nested ARHCF has a core diameter of $37 \mu \mathrm{m}$, surrounded by seven nested capillaries, with wall thicknesses of $\sim 406 \mathrm{~nm}$ and $\sim 630 \mathrm{~nm}$ for the outer and inner capillaries, as shown by Figure 3(a). This structure enables a low fiber loss at the NIR region, while preserves a high loss after $3 \mu \mathrm{m}$, therefore facilitating the cascaded rotational SRS by preventing the extra pump energy consumption with the vibrational SRS (see Figure 3(b)). At the output side of the nested ARHCF, the power of each individual Stokes line was measured by extracting them with a series of long-pass filters.
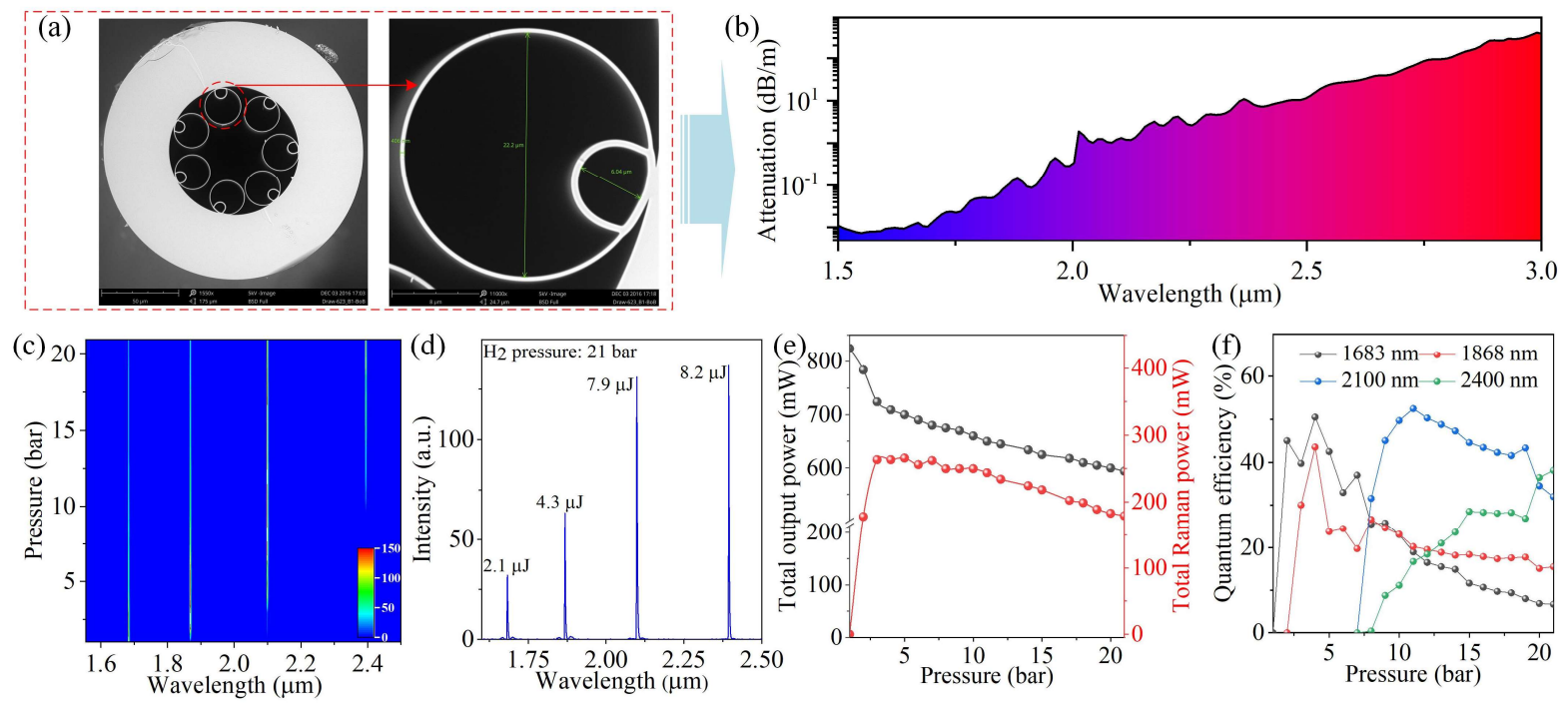

Figure 3. Multi-wavelength NIR Raman laser generation in $\mathrm{H}_{2}$-filled ARHCF. (a) SEM images of the nested ARHCF. (b) Simulated loss spectrum of the nested ARHCF. (c) Experimental result showing the evolution of the Raman laser spectrum as a function of $\mathrm{H}_{2}$ pressure. (d) Optical spectrum of the cascaded Raman fiber laser at 21 bar $\mathrm{H}_{2}$. (e) Total output power (black) and total power of all Stokes lines (red) versus pressure. (f) Calculated quantum efficiencies of each Raman Stokes line versus pressure.

The evolution of the measured Raman spectrum in terms of pressure was investigated at the maximum pump power and proper orientation of the quarter-wave plate, as presented in Figure 3(c). An optical spectrum analyzer (OSA) (Spectro 320, Instrument Systems) was used to record the spectra. Initially, only the first-order rotational Stokes line at $1683 \mathrm{~nm}$ was observed at the pressure of $\sim 1$ bar. Increasing the gas pressure increases the Raman gain coefficient of the steady-state Raman regime ${ }^{15}$ and suppresses the transient Raman regime ${ }^{16}$, which has a lower gain coefficient than the steady-state regime. As a result, the intensity of the first-order Stokes line first continuously increases until it is sufficient to serve as a new pump to generate the second-order Stokes line at $1868 \mathrm{~nm}$. Similarly, the third-order Stokes line at $2099 \mathrm{~nm}$ was formed from the second-order Stokes line and then increases in intensity and finally leads to the formation of a fourthorder Stokes line at $2394 \mathrm{~nm}$ when the pressure increases to $\sim 11$ bar. It can be seen that the emergence of new Stokes lines is always accompanied by a decrease in the intensity of the previous order Stokes line, indicating that the last order Stokes plays the role of the pump for the generation of the new Stokes line. Because the fifth-order Stokes line at $\sim 2.8 \mu \mathrm{m}$ could not be formed due to the high fiber loss of $105 \mathrm{~dB} / \mathrm{m}$, the intensity of the fourth-order Stokes line continuously increases with pressure up to $\sim 21$ bar, which is the maximum available pressure of the experimental setup. Figure 3(d) shows the measured spectrum at $21 \mathrm{bar}$, where the pulse energies are 2.1, 4.3, 7.9, and $8.2 \mu \mathrm{J}$ at $1683,1868,2099$, and $2394 \mathrm{~nm}$, respectively. Figure 3(e) shows the evolution of total power (includes all Stokes lines and residual pump) and total Raman laser power (includes all Stokes lines, but not the pump) as a function of gas pressure. It can be seen that the former continuously drops down as the gas pressure increases, indicating a energy transfer from the pump to the Raman laser Stokes lines. Meanwhile, the latter first quickly increases to a maximum value of $268 \mathrm{~mW}$, but then drops slowly again due to the saturation of the Raman gain coefficient with increase in $\mathrm{H}_{2}$ pressure ${ }^{15}$. This is mainly attributed to the low photon energy at longer wavelengths and the increased fiber propagation loss experienced by the higher-order Stokes lines at longer wavelengths. Figure 3(f) shows the quantum efficiency for each Raman Stokes line. The maximum quantum efficiencies at $1683 \mathrm{~nm}, 1868 \mathrm{~nm}, 2099 \mathrm{~nm}$, and $2394 \mathrm{~nm}$ are $50.5 \%, 43.5 \%, 52.5 \%$, and $38.1 \%$ respectively, corresponding to pulse energies of $18.5 \mu \mathrm{J}, 14.4 \mu \mathrm{J}, 14.1 \mu \mathrm{J}$ and $8.3 \mu \mathrm{J}$, respectively. One can see that the pulse energy of each Stokes line can easily reach the micro-joule level. To the best of our knowledge, this is the first time to achieve microjoule pulse energy over four cascaded Raman Stokes lines in such a broad spectral range. 


\section{CONCLUSION}

We summarized our recent works on gas-filled ARHCF lasers, including a SC generation spanning from $200 \mathrm{~nm}$ to $4 \mu \mathrm{m}$, and high energy infrared Raman lasers in single wavelength and multiple wavelengths. These results reveal the huge potential of the gas-filled ARHCF technology for laser emission from DUV to MIR, and provide diverse and powerful solutions for applications such as gas spectroscopy, imaging, etc.

\section{ACKNOWLEDGEMENTS}

This work is supported by the Danmarks Frie Forskningsfond Hi-SPEC project (Grant No. 8022-00091B), Multi-BRAIN project funded by the Lundbeck Foundation (R276-2018-869), ECOMETA (Grant No. 6150-00030B) and US ARO (Grant No. W911NF-19-1-0426). Abubakar I. Adamu is now with Lumenisity Ltd, United Kingdom.

\section{REFERENCES}

[1] Markos, C., Travers, J. C., Abdolvand, A., Eggleton, B. J. and Bang, O., "Hybrid photonic-crystal fiber," Rev. Mod. Phys. 89(4), 45003 (2017).

[2] Konorov, S. O., Fedotov, A. B. and Zheltikov, A. M., "Enhanced four-wave mixing in a hollow-core photoniccrystal fiber," Opt. Lett. 28(16), 1448-1450 (2003).

[3] Elu, U., Baudisch, M., Pires, H., Tani, F., Frosz, M. H., Köttig, F., Ermolov, A., St.J. Russell, P. and Biegert, J., "High average power and single-cycle pulses from a mid-IR optical parametric chirped pulse amplifier," Optica 4(9), 1024-1029 (2017).

[4] Mak, K. F., Travers, J. C., Hölzer, P., Joly, N. Y. and Russell, P. S. J., "Tunable vacuum-UV to visible ultrafast pulse source based on gas-filled Kagome-PCF," Opt. Express 21(9), 10942-10953 (2013).

[5] Cassataro, M., Novoa, D., Günendi, M. C., Edavalath, N. N., Frosz, M. H., Travers, J. C. and Russell, P. S. J., "Generation of broadband mid-IR and UV light in gas-filled single-ring hollow-core PCF," Opt. Express 25(7), 7637-7644 (2017).

[6] Adamu, A. I., Habib, M. S., Petersen, C. R., Lopez, J. E. A., Zhou, B., Schülzgen, A., Bache, M., Amezcua-Correa, R., Bang, O. and Markos, C., "Deep-UV to mid-IR supercontinuum generation driven by mid-IR ultrashort pulses in a gas-filled hollow-core fiber," Sci. Rep. 9(1), 4446 (2019).

[7] Smith, C. R., Moltke, A., Adamu, A. I., Michieletto, M., Bowen, P., Moselund, P. M., Markos, C. and Bang, O., "Low-noise tunable deep-ultraviolet supercontinuum laser," Sci. Rep. 10(1), 18447 (2020).

[8] Benabid, F., Knight, J. C., Antonopoulos, G. and Russell, P. S. J., "Stimulated Raman scattering in hydrogen-filled hollow-core photonic crystal fiber," Science 80. 298(5592), 399 LP - 402 (2002).

[9] Benabid, F., Couny, F., Knight, J. C., Birks, T. A. and Russell, P. S. J., "Compact, stable and efficient all-fibre gas cells using hollow-core photonic crystal fibres," Nature 434(7032), 488-491 (2005).

[10] Mridha, M. K., Novoa, D., Bauerschmidt, S. T., Abdolvand, A. and Russell, P. S. J. "Generation of a vacuum ultraviolet to visible Raman frequency comb in H2-filled kagomé photonic crystal fiber," Opt. Lett. 41(12), 28112814 (2016).

[11] Krupa, K., Baudin, K., Parriaux, A., Fanjoux, G. and Millot, G. "Intense stimulated Raman scattering in $\mathrm{CO}_{2}$-filled hollow-core fibers," Opt. Lett. 44(21), 5318-5321 (2019).

[12] Xiong, D., Bai, Y., Zuo, D. and Wang, X., "High-resolution continuous-wave coherent anti-Stokes Raman spectroscopy in a CO2-filled hollow-core photonic crystal fiber," J. Raman Spectrosc. (2021).

[13] Chen, Y., Wang, Z., Gu, B., Yu, F. and Lu, Q., "Achieving a $1.5 \mu \mathrm{m}$ fiber gas Raman laser source with about 400 kW of peak power and a $6.3 \mathrm{GHz}$ linewidth," Opt. Lett. 41(21), 5118-5121 (2016).

[14] Bischel, W. K. and Dyer, M. J., "Temperature dependence of the Raman linewidth and line shift for the Q(1) and $\mathrm{Q}(0)$ transitions in normal and para- $\mathrm{H}_{2}$, , Phys. Rev. A 33(5), 3113-3123 (1986).

[15] Hanna, D., Pointer, D. and Pratt, D., "Stimulated Raman scattering of picosecond light pulses in hydrogen, deuterium, and methane," IEEE J. Quantum Electron. 22(2), 332-336 (1986).

[16] Astapovich, M. S., Gladyshev, A. V, Khudyakov, M. M., Kosolapov, A. F., Likhachev, M. E. and Bufetov, I. A., "Watt-level nanosecond 4.42- $\mu \mathrm{m}$ Raman laser based on silica fiber," IEEE Photonics Technol. Lett. 31(1), 78-81 (2019). 
[17] Wang, Y., Dasa, M. K., Adamu, A. I., Antonio-Lopez, J. E., Habib, M. S., Amezcua-Correa, R., Bang, O. and Markos, C., "High pulse energy and quantum efficiency mid-infrared gas Raman fiber laser targeting $\mathrm{CO}_{2}$ absorption at $4.2 \mu \mathrm{m}$," Opt. Lett. 45(7), 1938-1941 (2020).

[18] Cao, L., Gao, S., Peng, Z., Wang, X., Wang, Y. and Wang, P., "High peak power $2.8 \mu \mathrm{m}$ Raman laser in a methanefilled negative-curvature fiber," Opt. Express 26(5), 5609-5615 (2018).

[19] Gladyshev, A. V, Kosolapov, A. F., Khudyakov, M. M., Yatsenko, Y. P., Kolyadin, A. N., Krylov, A. A., Pryamikov, A. D., Biriukov, A. S., Likhachev, M. E., Bufetov, I. A. and Dianov, E. M., "2.9, 3.3, and $3.5 \mu \mathrm{m}$ Raman lasers based on revolver hollow-core silica fiber filled by ${ }^{1} \mathrm{H}_{2} / \mathrm{D}_{2}$ Gas Mixture," IEEE J. Sel. Top. Quantum Electron. 24(3), 1-8 (2018).

[20] Benabid, F., Bouwmans, G., Knight, J. C., Russell, P. S. J. and Couny, F., "Ultrahigh efficiency laser wavelength conversion in a gas-filled hollow core photonic crystal fiber by pure stimulated rotational Raman scattering in molecular hydrogen," Phys. Rev. Lett. 93(12), 123903 (2004).

[21] Cui, Y., Huang, W., Li, Z., Zhou, Z. and Wang, Z., "High-efficiency laser wavelength conversion in deuteriumfilled hollow-core photonic crystal fiber by rotational stimulated Raman scattering,” Opt. Express 27(21), 3039630404 (2019).

[22] Adamu, A. I., Wang, Y., Habib, M. S., Dasa, M. K., Antonio-Lopez, J. E., Amezcua-Correa, R., Bang, O. and Markos, C., "Multi-wavelength high-energy gas-filled fiber Raman laser spanning from $1.53 \mu \mathrm{m}$ to $2.4 \mu \mathrm{m}$," Opt. Lett. 46(3), 452-455 (2021).

[23] Delfyett, P. J., Gee, S., Choi, M.-T., Izadpanah, H., Lee, W., Ozharar, S., Quinlan, F. and Yilmaz, T., "Optical frequency combs from semiconductor lasers and applications in ultrawideband signal processing and communications," J. Light. Technol. 24(7), 2701 (2006).

[24] Wang, Y., Li, J., Zhai, B., Hu, Y., Mo, K., Lu, R. and Liu, Y., "Tunable and switchable dual-wavelength modelocked $\mathrm{Tm}^{3+}$-doped fiber laser based on a fiber taper," Opt. Express 24(14), 15299-15306 (2016).

[25] Malitson, I. H., "Interspecimen comparison of the refractive index of fused silica," J. Opt. Soc. Am. 55(10), 12051209 (1965).

[26] Kitamura, R., Pilon, L. and Jonasz, M., "Optical constants of silica glass from extreme ultraviolet to far infrared at near room temperature," Appl. Opt. 46(33), 8118-8133 (2007).

[27] Travers, J. C., Chang, W., Nold, J., Joly, N. Y. and St. J. Russell, P., "Ultrafast nonlinear optics in gas-filled hollow-core photonic crystal fibers [Invited]," J. Opt. Soc. Am. B 28(12), A11-A26 (2011).

[28] Russell, P. S. J., Hölzer, P., Chang, W., Abdolvand, A. and Travers, J. C., "Hollow-core photonic crystal fibres for gas-based nonlinear optics," Nat. Photonics 8(4), 278-286 (2014).

[29] Stolen, R., "Polarization effects in fiber Raman and Brillouin lasers," IEEE J. Quantum Electron. 15(10), 11571160 (1979).

[30] Habib, M. S., Antonio-Lopez, J. E., Markos, C., Schülzgen, A. and Amezcua-Correa, R., "Single-mode, low loss hollow-core anti-resonant fiber designs," Opt. Express 27(4), 3824-3836 (2019).

[31] Poletti, F., "Nested antiresonant nodeless hollow core fiber," Opt. Express 22(20), 23807-23828 (2014).

[32] Frosz, M. H., Roth, P., Günendi, M. C. and Russell, P. S. J., "Analytical formulation for the bend loss in singlering hollow-core photonic crystal fibers," Photonics Res. 5(2), 88-91 (2017).

[33] Bischel, W. K. and Dyer, M. J., "Wavelength dependence of the absolute Raman gain coefficient for the Q(1) transition in H_2," J. Opt. Soc. Am. B 3(5), 677 (1986).

[34] Flusberg, A. and Holmes, R., "Polarization dependence of gain in stimulated Raman scattering," Phys. Rev. Lett. 58(20), 2039-2042 (1987). 